\title{
3D FEM model development from 3D optical measurement technique applied to corroded steel bars
}

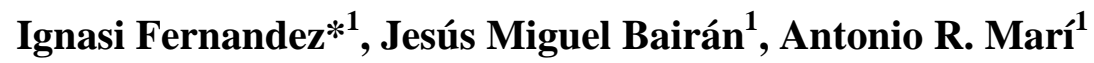

1 Department of Civil and Environmental Engineering, Polytechnic University of Catalonia, Jordi Girona, 1-3, Barcelona 08034, Spain.

*corresponding author: ignasi.fernandez-perez@upc.edu

Keywords: reinforcing steel; corrosion; 3D scanning; 3D FEM model; Tensile and fatigue properties

\section{Highlights:}

- 3D optical measurement for corrosion characterization and 3D finite element method (FEM) model development.

- Study of the local effects of pits on the corroded mechanical properties under cyclic and monothonic loads.

- Fibre model validation for fast simulation of mechanical properties of corroded steel bars.

\begin{abstract}
Understanding the mechanical effects of the corrosion pits on the steel surface requires an accurate definition of their geometry and distribution along the rebar. 3D optical measurement technique is used to obtain the outer geometry of artificially corroded bars tested under cyclic or monotonic loads. 3D FEM model development from the 3D scanning results were carried out in order to investigate the failure process and local effects on the pits, which are responsible of the variation of the mechanical properties in corroded steel reinforcement. In addition, a validation of a simplified model, which allows the mechanical steel properties determination given an estimated corrosion level, is presented. 3D models were convenient to observe and measure the local effects on the pits.
\end{abstract}




\section{Introduction}

Deteriorated structures can yield in an unexpected response under design loads. The steel susceptibility of being corroded under numerous conditions, as well as the structural effects of corrosion have been widely studied by many researchers [1-7]. Corrosion of steel reinforcement is described by a number of pits distributed along the steel bar, either generalised or pitting corrosion could be understood in this way [8-10] depending on the frequency and concentration of pits formed. Hence, what in first instance is an almost uniaxial stress state, it may turn into a multiaxial stress state in the surrounding of the pits and the critical cross-section. The study of corrosion in such a local way is relevant to deepen in the corrosion phenomenon and its effects on the steel bar [11,12]. Consequently, techniques such as 3D optical measurement of corroded steel bars are presented as powerful tools to study the aforementioned phenomena. With the application of this method, it is possible to analyse the local effects of steel corrosion produced on the bar by means of the outer surface description with a very high definition. 3D scanning, which is widely used in other fields such as industrial engineering, has not been commonly used in civil engineering. Since 3D scanning is a relative new technique and particularly its application to deteriorated structures and their different structural elements, a scarce number of studies involving 3D optical mesurement and its applications are available in the literature [13-16]. These studies are mainly focused on the pit characterization of corroded steel members using the cross-section definition and the pit distribution along the corroded bar. The use of this technique open a broad range of possibilities such as: statistical analysis of the pitting distribution, 3D solid modelling of the entire bar giving insight to local effects such as stress concentration and multiaxial stress distribution, among others.

An experimental work encompassing 12 two span statically indeterminate beams submitted to accelerated corrosion was presented by Fernandez et al. [17]. Corrosion levels between 9\% and $24 \%$ were reached. After testing the beams up to failure, steel bars were carefully extracted of the beams to perform the characterization of the corroded mechanical properties under monotonic and fatigue loads [9]. A symplified 2D mechanical model to assess the mechanical properties of steel bars, was extended by Fernandez et al. [10] to include both pitting and generalised corrosion phenomena, and further calibrated and validated with the experimental work presented by Fernandez et al. [9]. Finally, in this study, a 3D scanning technique applied to some of the extracted corroded steel bars is presented. A complete high-resolution description of the outer surface from different corroded specimens was obtained. The study encompassed 14 bars, which were scanned and afterwards divided into two sets to be tested until failure under fatigue and monotonic loading.

The first part of this research consisted in the 3D model development of the scanned specimens, with the purpose of reproducing the test condition and thereafter perform a comparison with the experimental data. To pursue this goal, DIANA [18] finite element software combined with the pre and post processor GiD [19] were used. The direct tensile and cyclic load test were both reproduced with DIANA. Additionally a fatigue model to identify the damage level of each element after every cycle was developed by means of an external subroutine. 
In the second part of the research work, the experimental results obtained were compared against the aforementioned cross-sectional model (2D) [10] in order to validate some of the hypotheses made in its formulation. Therefore, the geometry of the critical cross-section from the scanned bars, which was previously identified from the monotonic tests, is obtained. The Finite Element (FE) meshes generated with GiD [19] were introduced into the said model to obtain the corroded $\sigma-\varepsilon$ curves and afterwards compared the idealized pitted cross-section $\sigma-\varepsilon$ curves as well as the 3D model results.

\section{Corrosion procedure description. Members extraction, cleaning and preparation}

Steel bars were extracted from beams subjected to different corrosion degrees by means of induced corrosion methods [20-22]. The beams were cast incorporating in the mixture 4\% $\mathrm{NaCl}$ by cement weight, breaking the steel passive protective layer. A particular current density was applied to assure the desired corrosion degree. This was done through a DC power supply with an ammeter to monitor and fix the current intensity. The current direction was defined fixing the reinforcing steel as anode and the stainless steel bar disposed in the center of the specimen cross-section as the cathode. Each beam was reinforced with bars of two different diameters (10 mm and $12 \mathrm{~mm}$ ). Monotonic load tests were carried out in the continuous beams [17]. Finally, bars were carefully extracted from the non-critical section of the beams to perform the characterization of the corroded bars under monotonic and fatigue loads. The chemical compostition of the actual steel bars, B500SD [23], is described in Table 1 (see Table 2 for different EU denominations and standards and Table 3 for mechanical characterization); it should be mentioned that compositions can slightly vary between each steel casting depending on the original casting materials.

Using gravimetric methods, the loss in weight of the specimens was determined according to ASTM code [24]. A pressure sand blasting method was applied to remove both rust and bonded cement. In total 14 specimens were selected and scanned, covering corrosion rates from $9 \%$ to 22\% for $12 \mathrm{~mm}$ diameter bars, see Table 4 .

\section{3D optical measurement of the corroded bars}

The 3D scanning of the corroded bars was done by means of optical measurement. An industrial stereo device with two cameras of $5 \mathrm{MP}$ was used, Fig. 1. This technology requires fewer scans and delivers higher quality data even when complex geometries are scanned. The accuracy provided by the camera is $2.5 \mu \mathrm{m}$, which allows describing all the imperfections over the steel bar surface due to corrosion. A global coordinate system was set and referenced to the bar end. A correction of the measurement inaccuracies and data treatment were done using the post processing software Geomagic Wrap 2014 [25]. 


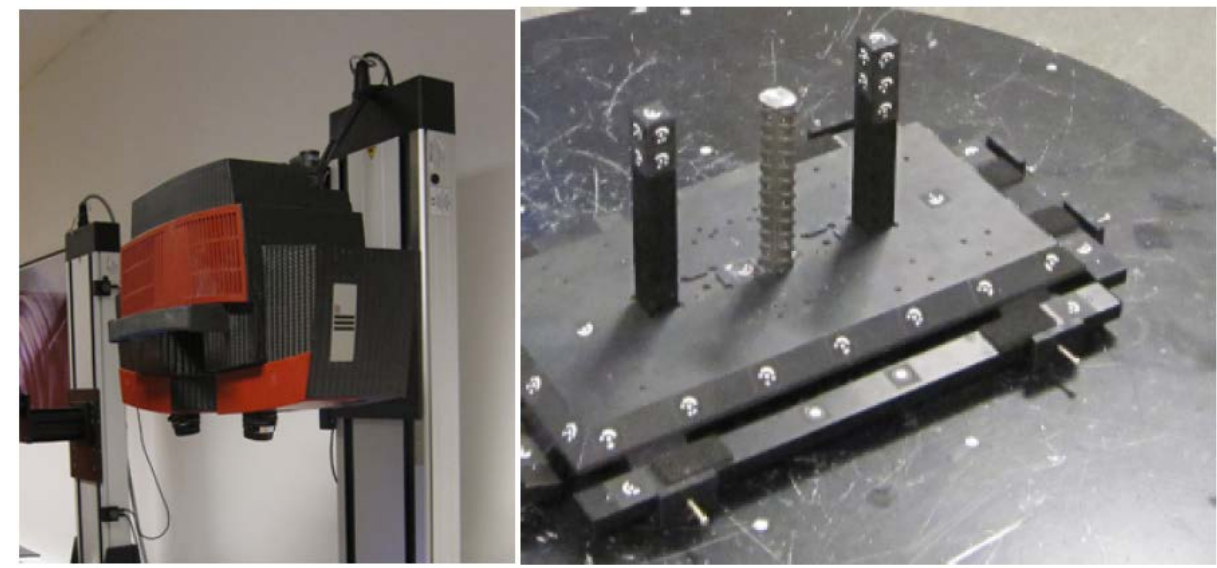

Figure 1. 3D optical measurement test setup for uncorrected bar

The outcome of the optical measurement was a very fine mesh of triangular surface polygons connected by nodes. The average size of the element was $0.005 \mathrm{~mm}^{2}$. The number of triangular elements in each scanning was between 700,000 and 1,100,000 elements, depending on the member length. The average specimen length was $320 \mathrm{~mm}$. Fig. 2 shows some specimens representing different corrosion degrees as well as an uncorroded specimen. The high resolution of the surface mesh allowed to define with great detail the pit dimension and, in particular, the pit depth and the pit length, which are the crucial parameters to describe the behaviour of corroded steel bars.

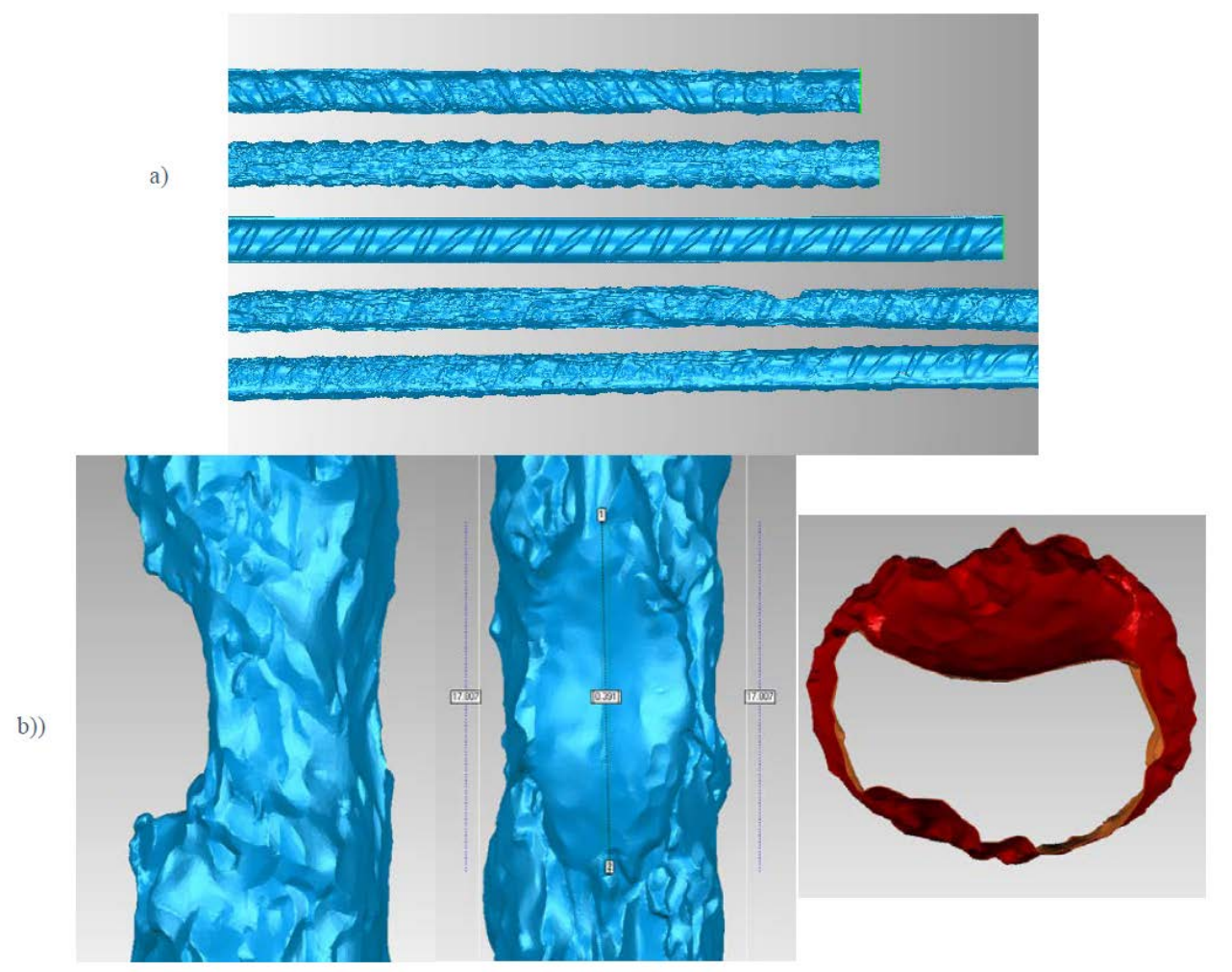

Figure 2. a) Scanned specimens representing different corrosion degrees and uncorroded specimen (in the middle). b)b) Critical cross-section definition $3 \mathrm{D}$ 
Fig. 2 depicts the critical pit of one of the scanned bars. The characterization and assessment of the pit geometry representing the pit length, pit depth, as well as the remaining cross-section were performed.

On the other hand, by substracting values of the geometrical parameters defining the corroded specimen from those of the same bar scanned when it was uncorroded, it would be possible to define the corrosion degree with a very good approximation.

\section{Cyclic load and tensile test}

\subsection{Test setup and execution}

The presented tests focuses on direct monotonic and cyclic tests of 14 corroded reinforcement bars extracted from the above mentioned beams, previously scanned with the novel 3D scan technique, see Fig 1 . The test were performed following the same methology presented by Fernandez et al. [9].

Reinforcing steel class B500SD was used in the monotonic and fatigue test for corroded and uncorroded specimens. This steel was produced following the manufacturing technique known as TEMPCORE ${ }^{\circledR}$. This technique consists on a severe tempering by applying pressure vapour of water on the bar's surface just after coming out of the lamination train. Hence the external temperature is reduced approximately from $800{ }^{\circ} \mathrm{C}$ to $400{ }^{\circ} \mathrm{C}$. Since the internal heat still exists in the bar's core, a further reheating takes place which helps to reduce residual stresses produced during the tempering. This process due to the variation of temperatures of the different zones produces heterogeneous material microstructures along the cross-section, Fig. 3.

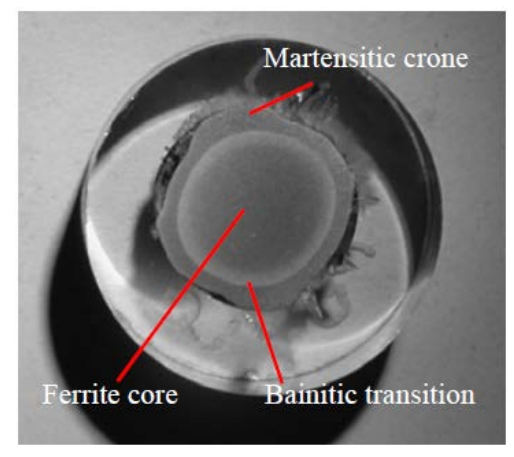

Figure 3. TEMPCORE® cross-section

\subsection{Monotonic test}

A total of 6 specimens were scanned and later tested under monotonic load. The tests were carried out following the code recommendations [26] on an INSTRON 8803 Universal Testing machine.

The specimens utilised for monotonic testing had between $310 \mathrm{~mm}$ and $320 \mathrm{~mm}$ length. The tested free length was for all the specimens $170 \mathrm{~mm}$ letting 70/75 mm length for each clamp. Monotonic tests were conducted using displacement control until specimen failure. The load was applied directly to the bar controlled by the load cell placed on the top of the hydraulic jack. The load was applied until specimen failure. The total displacement, as well as the 
longitudinal deformation, which was measured using a displacement transducer, were registered too.

Fig. 4 described the $\sigma-\varepsilon$ behaviour of the corroded members. All the mechanical properties were affected by corrosion. A significant reduction of the yielding stress, as well as ultimate stress, was observed.

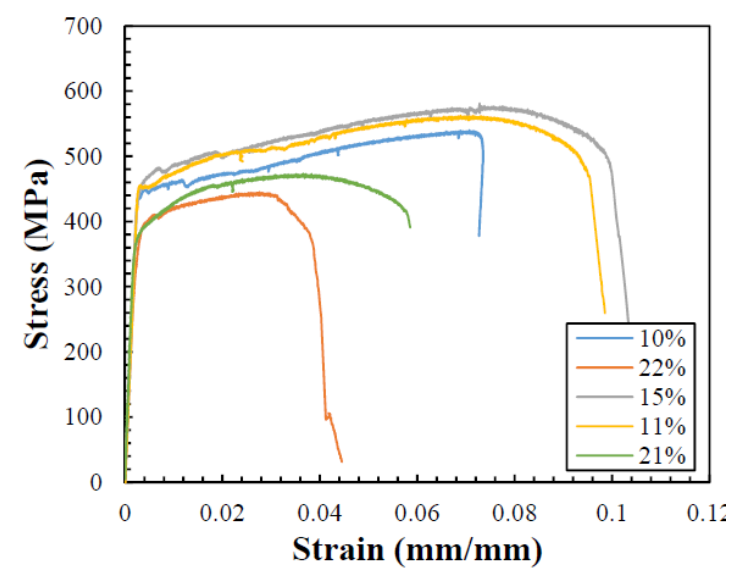

Figure 4. $\sigma-\varepsilon$ behaviour of tested corroded specimens

\subsection{Fatigue test}

In total 8 specimens were scanned and later tested under cyclic loads. The specimen's free length was the same as in the monotonic tests of the previous section. Fatigue tests were conducted under load control instead displacement control in order to provide a fixed range of cyclic load. Stress levels defined for each test, and the corresponding loads to be applied, were referred to the intact bar geometry, which is independent of the degree of corrosion.

Two different stress ranges were investigated, 3 specimens for each stress range, namely 200 $\mathrm{MPa}$ and $300 \mathrm{MPa}(\Delta \mathrm{S}=\mathrm{Smax}-\mathrm{Smin})$. A non-zero minimum stress (Smin) was used to assure that no accidental compression was applied to the bar, thus avoiding the risk of buckling. The maximum stress (Smax) was always lower than $0.6 * \mathrm{f}_{\mathrm{ym}}$, being $\mathrm{f}_{\mathrm{ym}}$ the yield stress of the reference uncorroded bar. According to current design practice [27,28], below this threshold there is no influence of the stress level on the fatigue life. However, due to the reduction of volume of the corroded specimen and irregular stress distribution, it is likely that the actual stress might have locally exceeded that value. This effect is to be investigated in the following sections.

The applied load range for corroded specimens was the same as for uncorroded samples, taking into account the uncorroded nominal diameter, see eq 1.

$$
\text { (1) } \Delta S=S_{\max }-S_{\min }=\frac{F_{\max }}{A_{0}}-\frac{F_{\min }}{A_{0}}
$$

Where $S_{\text {max }}$ and $S_{\text {min }}$ are the maximum and minimum applied stresses, $F_{\text {max }}$ and $F_{\text {min }}$ the applied load on the bar calculated by considering the nominal uncorroded bar area, $A_{0}$. The load was applied following sinus shaped waves at a frequency of $15 \mathrm{~Hz}$. 
Table 5 shows the results of the performed fatigue tests, including the fatigue life value and the corrosion degree of the tests carried out.

\section{3D numerical model}

A 3D model was developed based on the transformation of the triangular outer surface mesh from the 3D scan results into a solid tetrahedral elements mesh. To proceed with the conversion, a data post-processing of the initial mesh was performed. First, a treatment to the surface mesh was done aiming at reducing the initial number of elements at the surface. A surface smoothing procedure was performed to remove spikes in the pits and badly shaped elements making possible the volume definition. Despite the remeshing procedure, the details of the initial geometry were preserved. Once the solid volume was created using the preprocessor GiD [19], it was possible to create a nurb surface from which the tetrahedral solid mesh was generated. Fig. 5 describes the schematic process performed from the 3D scan file (STL format) to the final 3D solid mesh.

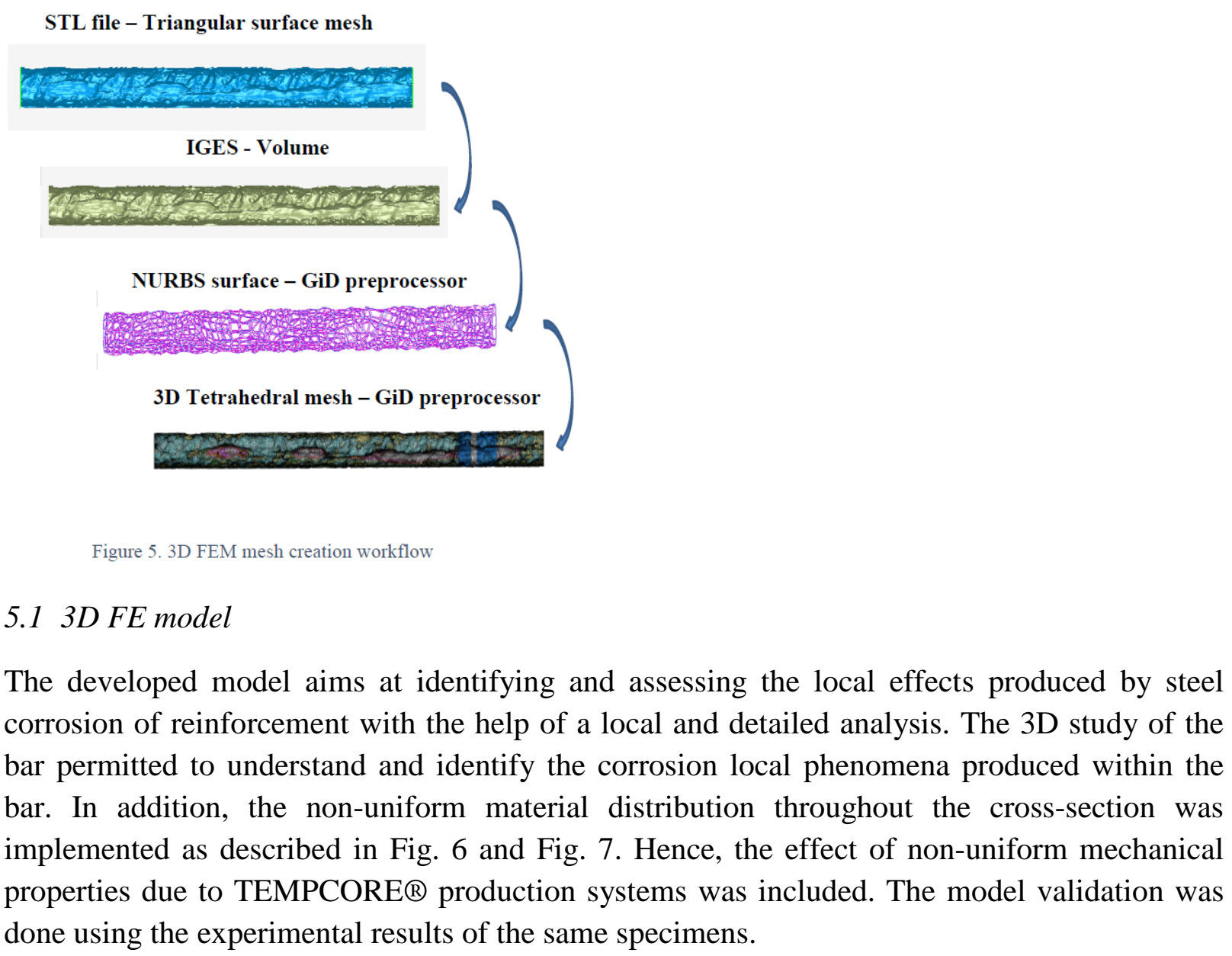




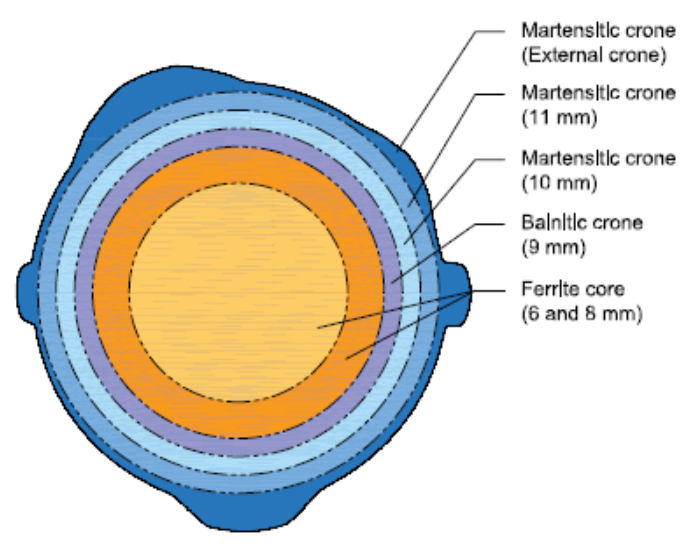

Figure 6. Ferrite inner core, bainitic transition and martensitic crown up to whole bar (typical non-uniform material distribution of TEMPCORE ${ }^{\circ}$ production system)

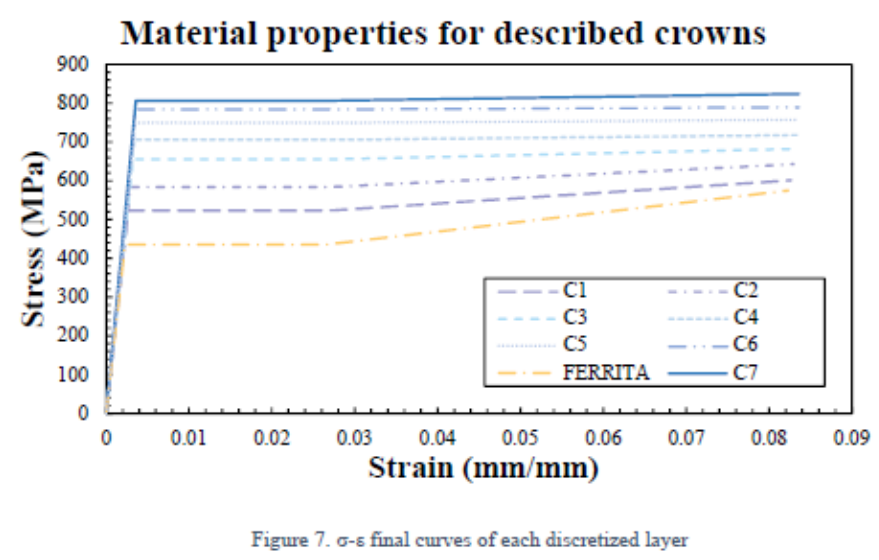

\subsection{Load and boundary conditions}

Boundary conditions were defined to reproduce the aforementioned test. One side of the bar was modelled constraining the displacements in the longitudinal direction. One additional node had constrained the displacements in the other two directions as mesh stabilizer, see Fig. 8. The other bar side was left unfixed, and the load was directly applied onto that face by means of displacement control or load control, depending on the simulation type. Load steps were used for fatigue simulations whereas displacement control was defined for tensile tests. 

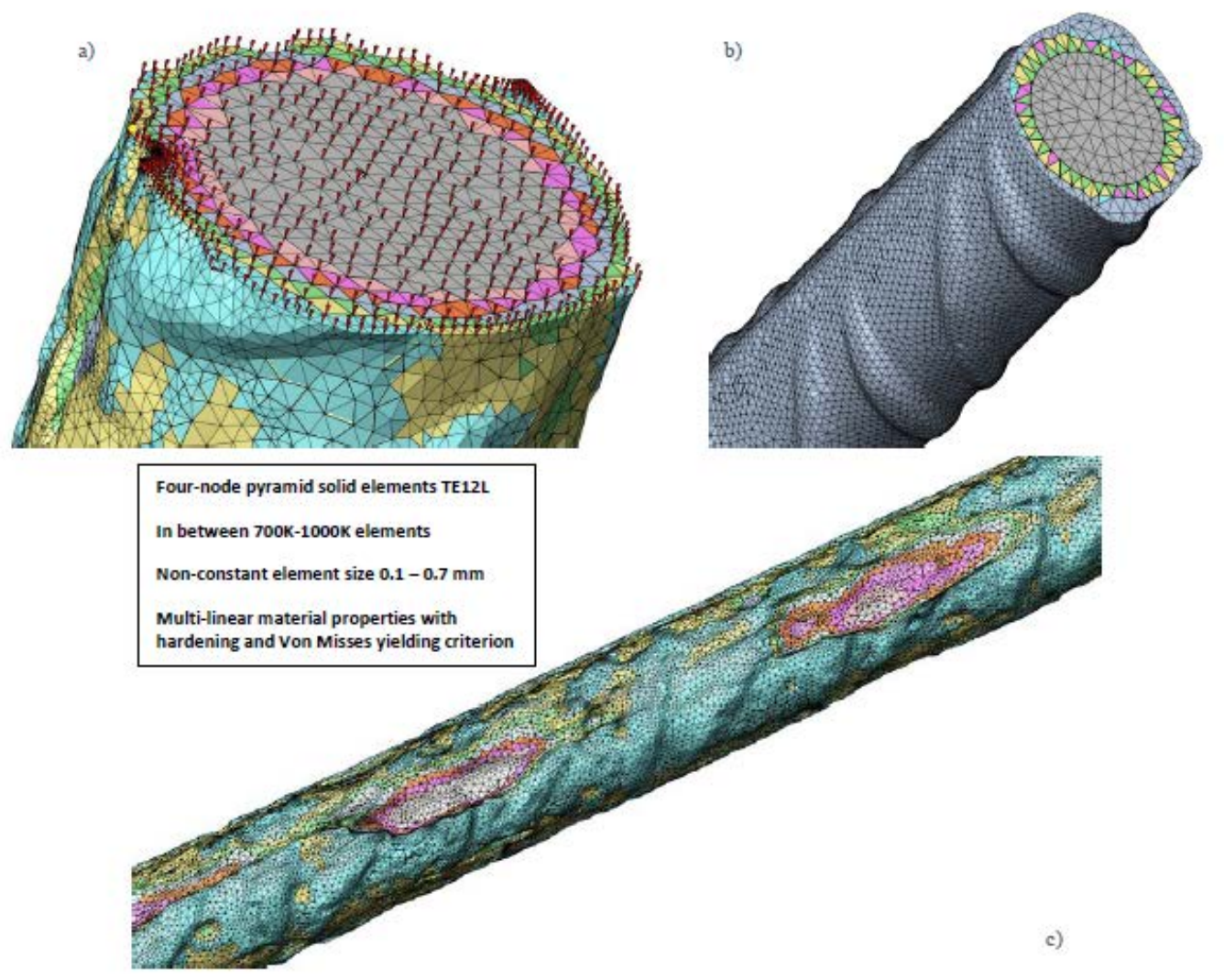

Figure 8. 3D FEM mesh and material data distribution. a) Corroded specimen; boundary conditions in the end support. b) Uncorroded specimen. c) General view of the mesh (each color represents one material layer)

\subsection{Element types and material definition}

3D tetrahedral elements (TE12L) were used for the steel bar definition. The bar was discretized with a significant number of 4 node tetrahedral elements. The models had an average of 800,000 elements.

The material properties of the steel elements were defined according to experimental data [10]. By performing monotonic tests to uncorroded specimens with different cross-section reductions, the different $\sigma-\varepsilon$ curves were obtained describing the variation of the mechanical properties throughout the steel cross-section. The reduction of cross-section was performed using a milling machine. Different crown thicknesses were removed from each specimen using a diamond tip. The actual diameter was checked using a Vernier calliper to know the exact remaining cylindrical steel area. The diameters tested corresponded to the ones described in Fig.6. By post-processing the obtained data, applying the product-summation series described in Eq. 2, the material properties for each particular crown could be obtained, see Fig. 7. In this figure is possible to see the descritazation of the cross-section in martensitic/bainitic layers about $0.25 \mathrm{~mm}$ thick plus the ferrite core. The values of the material properties of the crowns in between the tested diamaters were linearly interpolated from the test resaults. Then 7 crowns to discritaze the material in between the ferrite core and the outer bar surface plus the inner ferrite core were defined. The final yielding and ultimate stress values for the different layers, both the tested and the interpolated ones, are shown in Table 6.

(2) $\sum A_{i} \cdot f_{y_{i}}=\bar{A} \cdot \overline{f_{y}}$ 
Where $\bar{A}$ corresponds to the total uncorroded area, $\bar{f}_{y}$ to the yielding stress of the uncorroded bar and $A_{i}$ and $f_{y_{i}}$ the same aforementioned parameter for each tested diameter. An independent subroutine to assign the $3 \mathrm{D}$ model element material properties was developed, which took the different element nodes coordinates and calculated the 3D tetrahedral element centre. The material properties of each element were assigned according to the distance of the element's centroid to the centre of the bar, following Fig. 6 and Fig. 7. The centroid determination for the corroded bars was done overlapping the identified most uncorroded cross-sections of the bar with the corroded cross-section. When the bar did not present any uncorroded zone to work with an adjustment considering uniform corrosion was done also overlapping the specific crosssection with the reference bar cross-sections. It has to be noticed that without the scan of the same bar before corrosion, it was not possible to define the centroid with more precision. The final meshes with the typical material data distribution are shown in Figs. 8a-c, for a corroded specimen and an uncorroded specimen, where each colour represents a different material layer. The incorporation of the non-homogeneous material distribution data described the TEMPCORE $^{\circledR}$ material distribution effects in the developed 3D model.

\subsection{Fatigue model development}

DIANA [18] software package allows for fatigue analysis only on linear elastic regime. However, since the corrosion procedure reduced the steel cross-section due to pitting distribution, non-uniform stress distribution throughout the cross-section including possible local yielding was expected. Hence, non-linear analysis was necessary to correctly assess the fatigue life of corroded members. That requirement motivated the development of an external fatigue life model to allow considering plastic behaviour in local zones through a series of static analyses of each stress level, which are then analysed by the script to determine the number of elements failing in each cycle, as described in the following.

For every pair of maximum and minimum applied loads, the corresponding stresses were obtained considering the nonlinear states in the specimen using the non-linear DIANA analysis. From those values, the mean stress value and range were determined for each finite element. A modification of the reference fatigue curve to consider these mean stress and range values was done, by modifying the $S_{\text {ref }}$ value. The reference fatigue curves are represented in Fig. 9a. The main material characteristic values used are described in Table 7. Palmgren-Miner rule, Eq. 3, was used to define the damage state of each element after every load step (j), Fig. 9b.

$$
\text { (3) } \sum_{j}^{1} d_{i}=\frac{n}{N_{j}}
$$

Where $N_{j}$ is the number of cycles resisted for stress range $j, n$ is the number of cycles applied, and $d$ is the damage index for the element $i$. The damage index was accumulated during every load step. When the element reached a damage index equal to 1, it was considered broken and it was removed from the model for the next steps. This process was repeated in a recursive manner until the last group of elements were not able to resist the applied load. At this point, the total number of applied load cycles $(n)$ is stated as the fatigue life value. 

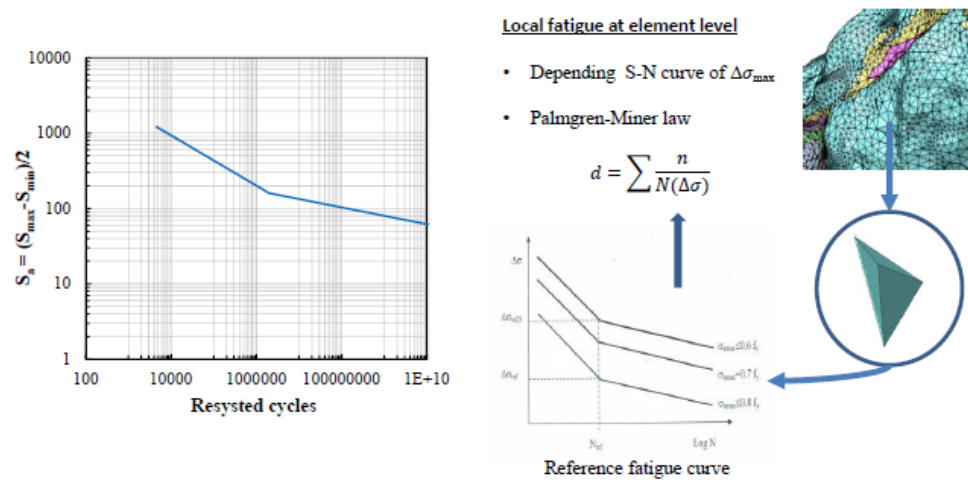

Figure 9. a) Reference fatigue curve [16,1]], b) Fatigue treated at element level

In the video Appendix A - Video 1, the fatigue model developed applied first to an homogeneous and smooth steel cylinder with a notch in the middle of the length is presented. A reference example in a simpler case without ribs or corrosion pits is showed. The video depicts the evolution of broken elements through a simulated fatigue test. The deterioration of a critical region can be observed, resulting in less effective area in that zone. Enventually, global failure takes place when the remaining area of the bar is not capable of resisting the applied load.

Fig. 10 shows a detailed flowchart of the developed fatigue model, including all the phases and assessments performed to each element until the fatigue life value is reached.

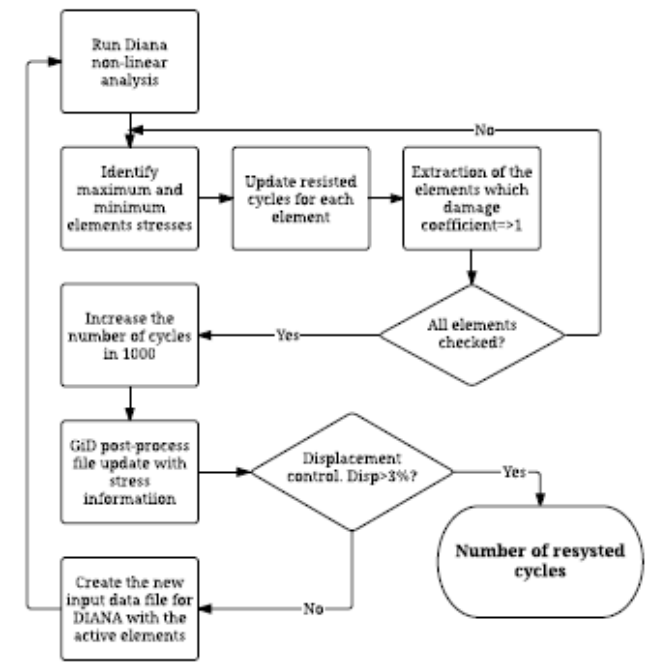

Figure 10. Flowchart fatigue developed model

\subsection{D model results}

\subsubsection{Fatigue results}

In Table 5 the numerical results of the 3D model presented here are described. In the same table a comparison between the experimental and the numerical data by means of the ratio $\mathrm{LN}\left(\mathrm{N}_{\text {test }}\right) / \mathrm{LN}\left(\mathrm{N}_{3 D \_m o d e l}\right)$ is showed; where $\mathrm{N}_{\text {test }}$ is the test resisted cycles and $\mathrm{N}_{3 \mathrm{D} \_m o d e l}$ are the $3 \mathrm{D}$ model resisted cycles. In addition, the mean value $(\mu)$, the standard deviation $(\sigma)$ and the COV for such values are presented. As observed the model adjusted well, although higher corrosion levels presented worse agreement. 
Specimen FT-03 is presented as an example of the capabilities of the developed 3D model from the 3D scan bar. This specimen achieved 11\% of corrosion degree and resisted 267,542 cycles in the experimental test. The numerical results predicted 287,322 cycles before failure, showing good agreement with the experimentation. Fig. 11 shows different output data from the 3D model. Surface increment stresses, total deformation, and the number of broken elements are depicted. As described in Fig. 11, the increment of stresses affected more the zones between ribs, as them due to geometrical effects took less stresses. Small red zones, indicating very high stress ranges are also described around the tips due to the stress concentration effect. In Fig. 11, the broken elements can be seen distributed along the entire specimen and not exclusively in the failure zone. Nevertheless, the failed cross-section presented the highest concentration of broken elements, due to the applied cycles. In the video Appendix A - Video 2 similar analysis to the model of a corroded ribbed bar is presented. It is evident that due to the increased number of irregularities produced by the pit distribution, more zones which involve broken elements are observed (multiple notches along the member). Despite the damage is more distributed along the member, the critical pit zone ends up describing the bar failure with higher amount of broken elements.

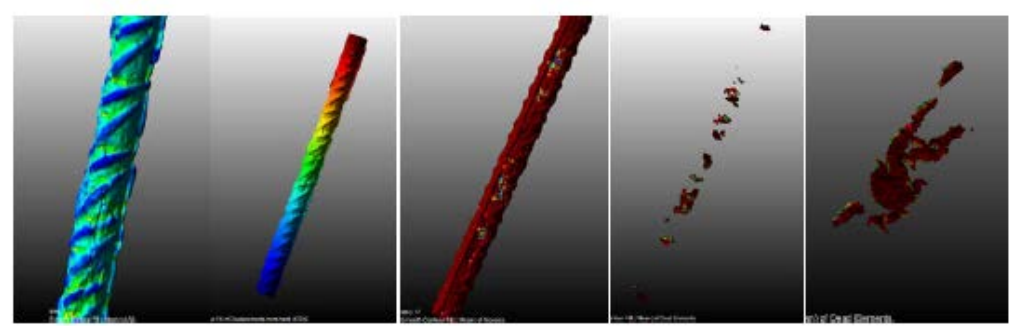

Figure 11. From left to right, stress distribution, total displacement, number of cycles resisted, dead elements whole bar and dead elements in the critical cross-section zone

\subsubsection{Tensile results}

A comparison between numerical and experimental results is presented in Figs. 12a-b. Fig. 12a shows an uncorroded specimen. Good agreement between the uncorroded experimental and presented model was obtained up to the maximum load; afterwards, the model stops without entering into the softening branch of the load-strain curve, since, as described in Section 5.3 and Fig. 7, the material models for each layer were considered as bi-linear without softening branch. The model continues until the ultimate strain defined in the material model is reached. Both the yielding stress and the ultimate stress were well captured; the error in the predicted yield stress was $3 \%$ with respect to the experimentation. It is evidenced that the uncorroded bar presents a clear yield plateau and strain hardening branch; while in the corroded, besides a noticeable reduction of the bar's capacity, the yield plateau is lost. 

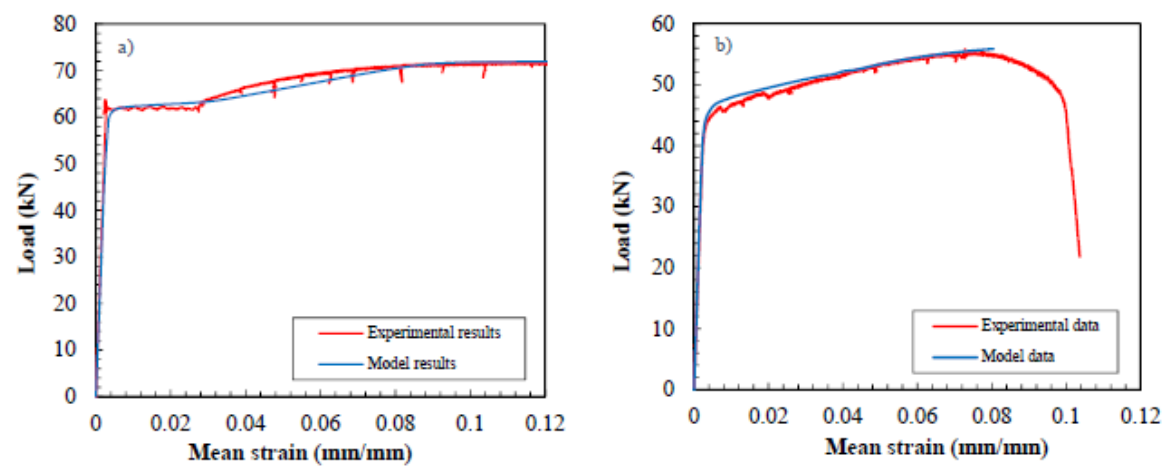

Figure 12. $\sigma-\varepsilon$ experimental and model results. a) Uncorroded specimen, b) Corroded specimen

Fig. 13 shows the different mechanical effects produced by the presence of pits along the specimen length. The cross-sections showed in Fig. 13, which represents two different views of the failure cross-section, were clearly subjected to local bending due to a displacement of the centre of gravity because of the pits. The mentioned effect leads to differences of stress, for instance in the ferrite core, approximately about $20 \%$ between the maximum and the minimum value. In spite of the different material properties, since the implemented material model slightly hides such effect, stress concentration due to the pits can also be observed. In Fig. 13, the non-uniform distribution of stresses along the specimen is also shown. For instance, the ribs of the bar presented lower stress levels than the remaining parts of the bar as consequence of geometrical effects on the cross-section. In general, the bars showed a multi-axial stress behaviour with non-uniform stress in the different cross-section. Following the different model outputs, it is possible to state that the local bending, due to the displaced centre of gravity, has a big influence in the final behaviour.

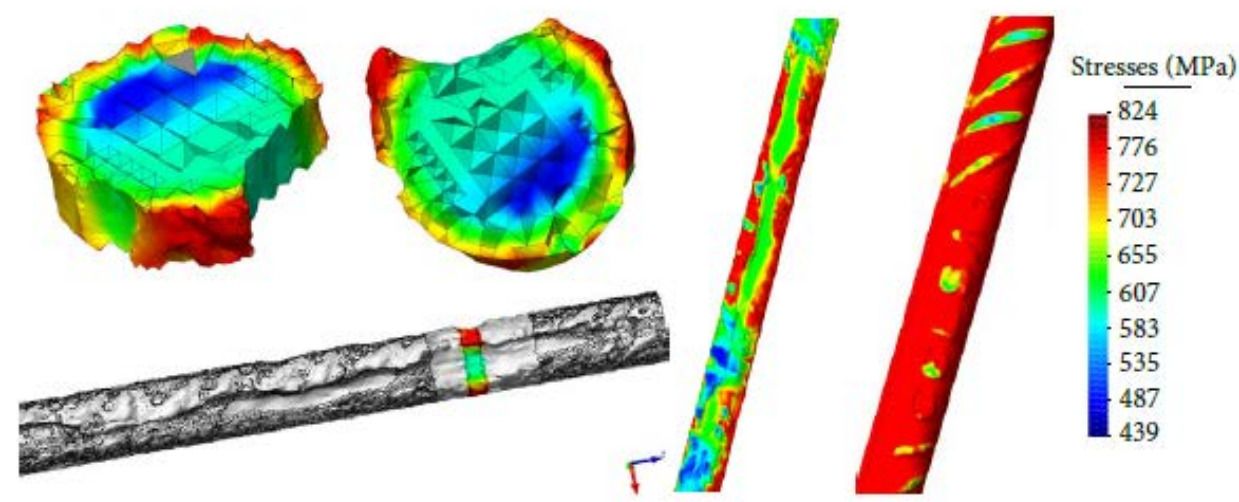

Figure 13. 3D FEM model outputs for tensile test. Mechanical effects of pits along the steel bar. Critical cross-section and whole bar

\section{FATSEC-Cor model validation. Critical cross-section definition}

The validation of the model FATSEC-Cor [10], for the fast assessment of mechanical properties of corroded bars, was carried out using the 3D scan results presented above. The model is based in a sectional fibre analysis accounting for the non-linear material properties of steel with mixed isotropic-kinematic hardening, effects of stress concentration in the region of the pit tip, local bending produced by translation of the bar's centroid when the pit corrosion is present, fatigue damage, among other effects. This model was used in previous studies to 
obtain the mechanical and fatigue properties of TEMPCORE® bars produced in rolls, in order to account for the effects of winding and unwinding of the rolls [29].

The experimental database presented in [9], with 142 fatigue tests and 40 monotonic tests, was used for calibration and validation purposes. A critical cross-section, where the bar would most probably fail, was defined by means of the characteristic pit definition, see Fig. 14. Fig. 15 describes the dimensionless model pit depth divided corroded diameter relationship with respect to the corrosion degree, once the model was calibrated. The final expression used to relate the characteristic critical pit with the corrosion level is also described. The upper (95\%) and lower (5\%) bounds of the model are depicted in the same figure, which indicated the most and the least damaged cross-sections for an specific corrosion degree, following the normal statistical distribution described in Fernandez et al. [10].

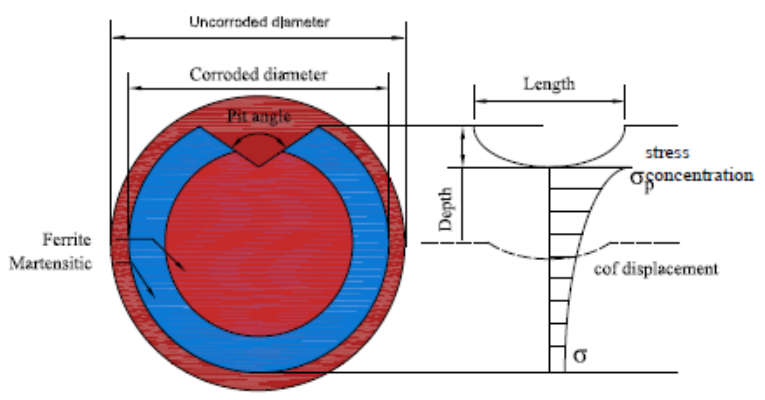

Figure 14. Pit effect in the steel cross-section: stress localization and local bending moment. Pitt definition in the model

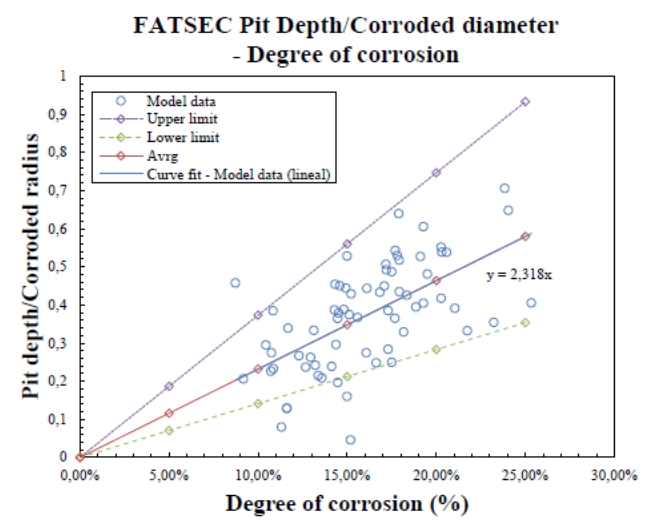

Figure 15. Relationship between degree of corrosion and the quotient pit depth - corroded bar diameter. Upper

and lower limits defined by the statistical model.

In this section a comparison between the above idealized critical cross-section and the real critical cross-section obtained from the 3D scan was performed. In addition, a comparison between the experimental data and the numerical results from the model was carried out. The model results were described using two hypotheses, the idealized cross-section previously described, Fig 14, and the real cross-section defined by means of the 3D scan. The results of the performed 3D model are also presented.

\subsection{Actual geometry of the critical cross-section}

Using the scanned data, it was possible to obtain the actual contour of the critical cross-section before failure. The steel area in the cross-section as well as an exact cross-section shape were, 
therefore, computed. Fig. 16 depicts the contour the critical section of four specimens through the scanned geometry.
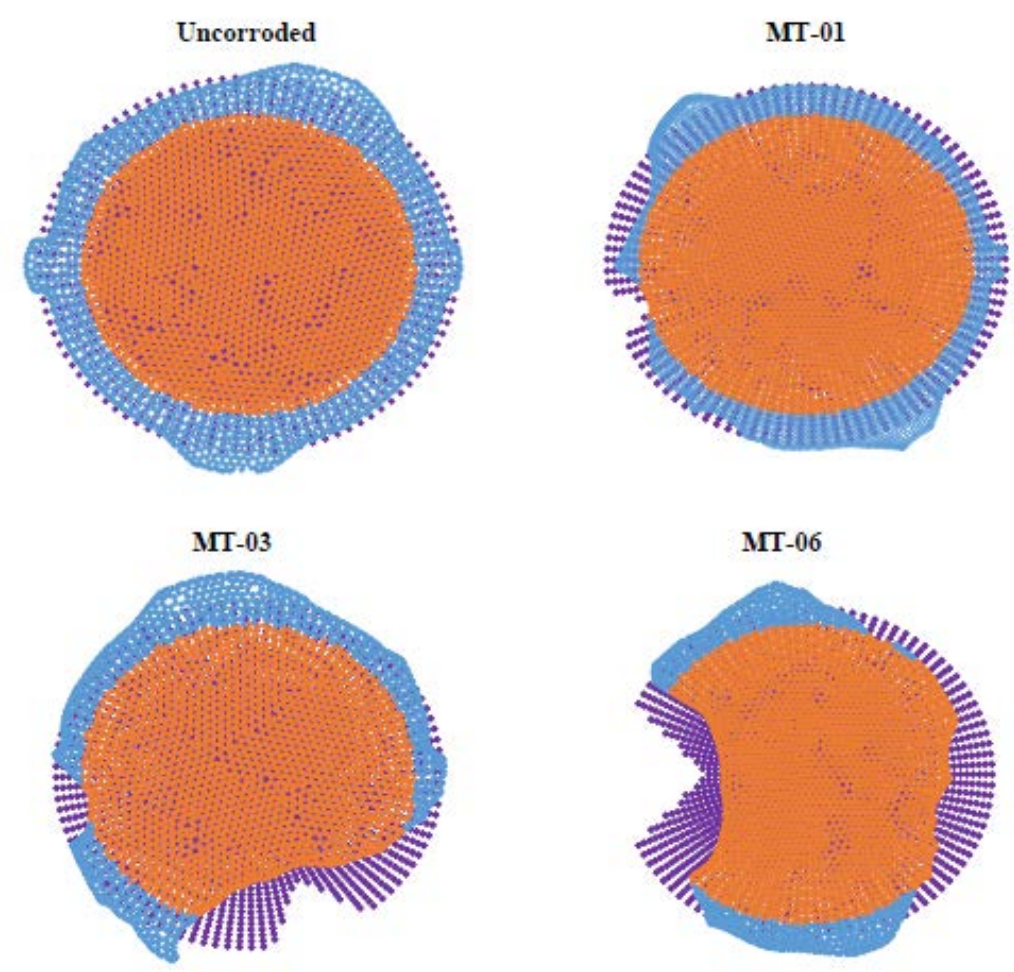

Figure 16. Real cross-section from 3D scanning and idealised cross-section defined by model FATSEC-Cor [10]. Each dot in the cross-section represents de c.o.g. of the triangular generated mesh. Each element is considered as a single fibre with the corresponding area of the triangle. In orange the ferrite elements and in blue the martensitic ones. In purple the idealised cross-section.

\subsection{Mesh generation and material definition}

FATSEC-Cor model used a fibre FE mesh discretisation of the cross-section based on GiD to perform the bar capacity calculations. Triangular elements were used to define the entire crosssection. The average number of elements used for its definition was around 5000 elements. The average value of the triangle maximum size was $0.15 \mathrm{~mm}$. Fig. 16 shows the centre of gravity of the generated mesh's triangles. The material, in orange and blue, and the area of the element is assigned to each dot.

A variation of the material assignation subroutine developed for the presented 3D model was implemented to define the material data of the described cross-sections. According to Bairan et al. [29], a two phase material discretization was considered in the model; the ferrite inner core and the martensitic external crown. The latter was defined using an average of the material properties corresponding to all the outer crones. Fig. 16 describes the mesh discretization of the bars' cross-sections for both the model hypothesis average pit depth consideration and the real cross-section, with the bilayer material assumption.

\subsection{Idealised pitted cross-section validation}

As observed in Fig. 16, the higher the corrosion in the cross-section, the more differences were observed between the actual and the idealized geometry. In all cases, the actual cross-section 
presented a larger area reduction than the idealized one. Moreover, it should be pointed out that while the actual distribution of pits varies along the bar length length and more than one pit may occur in a single cross-section, the fast assessment model FATSEC-Cor simulates the phenomenon with one equivalent pit, as observed in Fig. 14 and Fig. 16.

\subsection{Comparative analysis of the results}

Fig. 17 compares the results for the different cross-section investigated. It may be observed that using the mean depth of the equivalent pit depth, FATSEC-Cor model produce good agreement with the experimental results in terms of yielding strength and ultimate capacity. However, when the actual geometry of the critical cross-section is used in FATSEC-Cor the capacity is consistently less than in the experimental test.
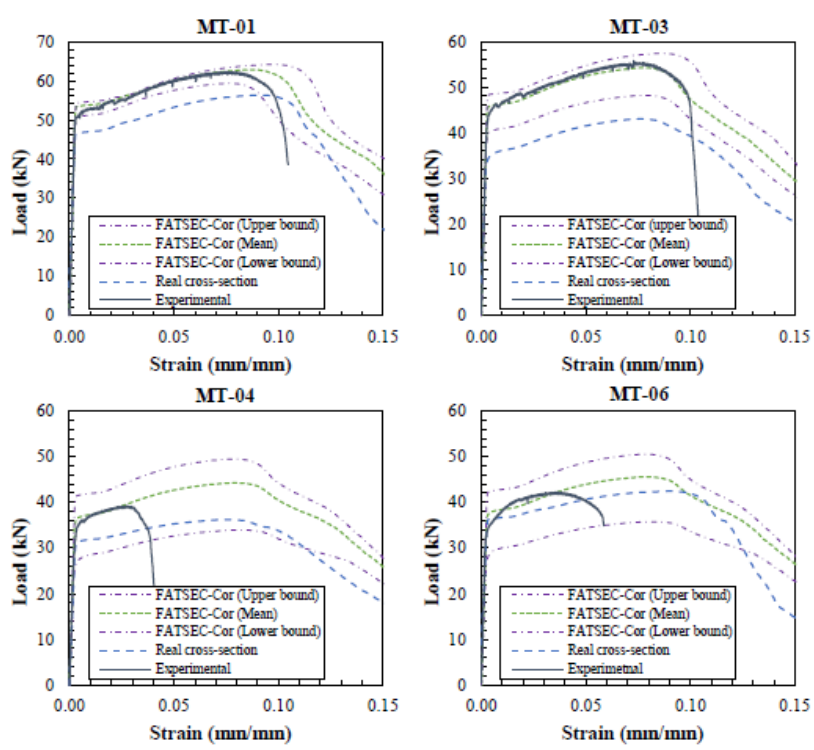

Figure 17. Result comparison. FATSEC-Cor (Upper, Lower and Mean pitting depth idealised cross-section and 3D scan cross-
section). Experimental results. In order MT-01 specimen, MT-03 specimen, MT-04 and MT-06

The observed differences may be explained by considering the reduced sectional area of the 3D model with respect to FATSEC-Cor, on one hand, and the influence of 3D stress distributions in the surrounding of the pit produced by the geometry irregularities. The actual geometry of the corroded bar may produce multi-axial stress states in the overall bar that are not considered in FATSEC-Cor model, as the latter only accounts for longitudinal stresses. This effect is investigated in the next section.

\subsubsection{FATSEC-Cor and 3D model results comparison}

A detailed analysis of the 3D results around the critical pit was carried out to confirm the hypothesis that multi-axial stress-strain state plays a relevant role in the ultimate capacity of the bar. The different graphical outputs of the stress level in the section that was used in FATSECCor, are shown in Fig. 18. In Fig. 18a, the stresses using the Von Mises criterion are represented, eq. 4 . On the other hand, the uniaxial stresses in the longitudinal direction of the bar at the same ultimate step in Fig. 18b. It may be observed that points with high 3D stress states, the longitudinal component of the stress may be higher than the uniaxial yielding strength. 


$$
\text { (4) } \sigma_{v}=\sqrt{\frac{1}{2}\left[\left(\sigma_{1}-\sigma_{2}\right)^{2}+\left(\sigma_{2}-\sigma_{3}\right)^{2}+\left(\sigma_{3}-\sigma_{1}\right)^{2}\right]}
$$

The previous analysis provides an explanation of why the bare critical section has lower capacity than the full bar, where 3D stresses may take place. Therefore, the ultimate capacity is not controlled only by the section with less area but it is rather a characteristic of the region surrounding that point. Hence, in the following, the FATSEC-Cor analyses of the measured geometry are repeated using an average geometry of a region of one diameter length centred in the critical cross-section, Fig. 19.
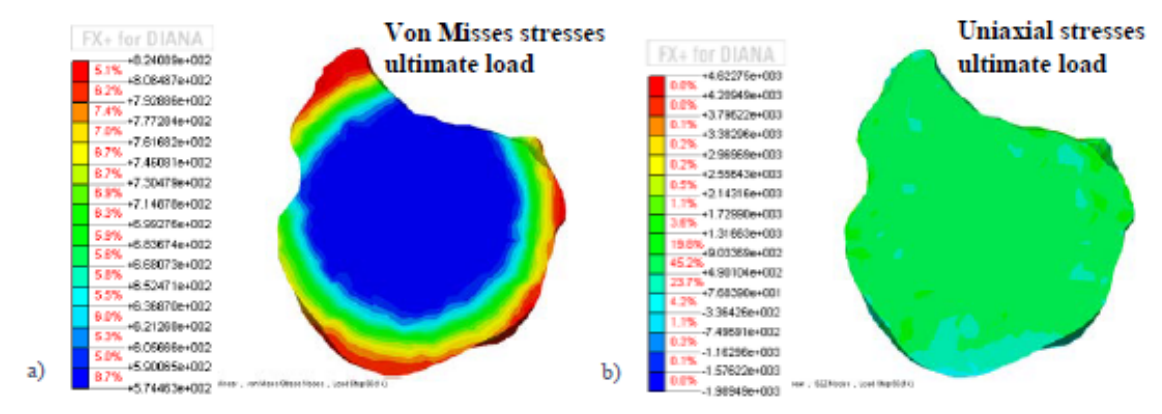

Figure 18. Multi-axial stress behavior of corroded specimens. Ultimate stress step
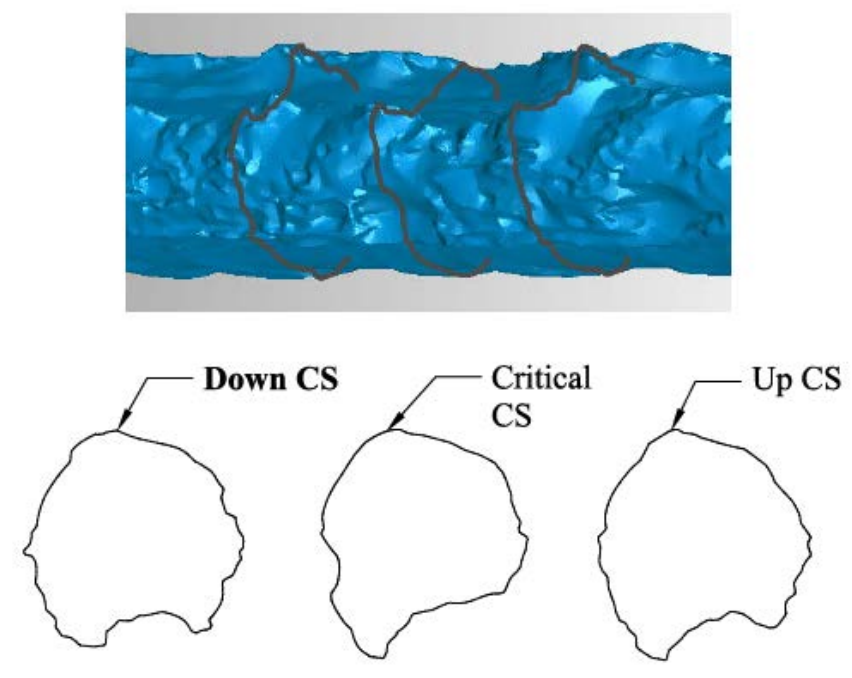

Figure 19. Real critical cross-section in the middle and cross-section defined into $65 \mathrm{~mm}$ up and down from the critical CS

It may be observed in Fig. 20 that the average critical cross-section adjusted better to the idealized pit hypothesis used by FATSEC-Cor, showing similar areas and pit depth than in Fig. 16. The numerical result of the average critical cross-section is described in Fig. 21. A good agreement between the experimental data, the FATSEC-Cor results using the average area as well as the presented before 3D model results is described. Therefore, the multiaxial stress state in the critical section can be reasonably approximated by the response of a uniaxial loaded average geometry along a certain length. An averaging length equals to the bar's diameter seems adequate. 

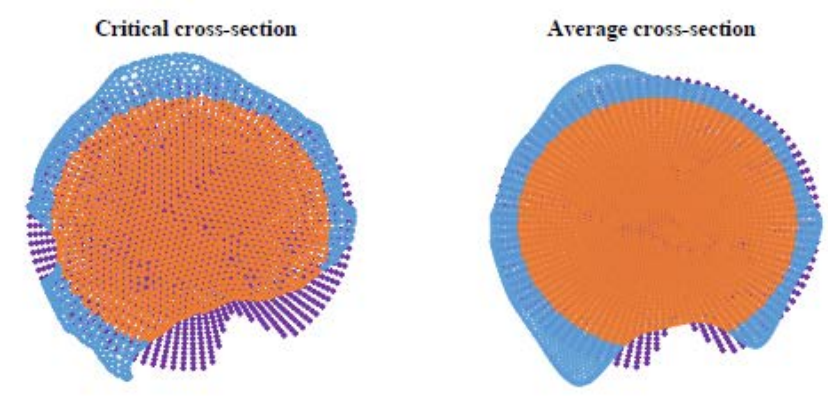

Figure 20. Real critical cross-section compared to idealized pitted cross-section - Left. Average critical cross-section compared to idealized pitted cross-section - Right (MT-03 specimen)

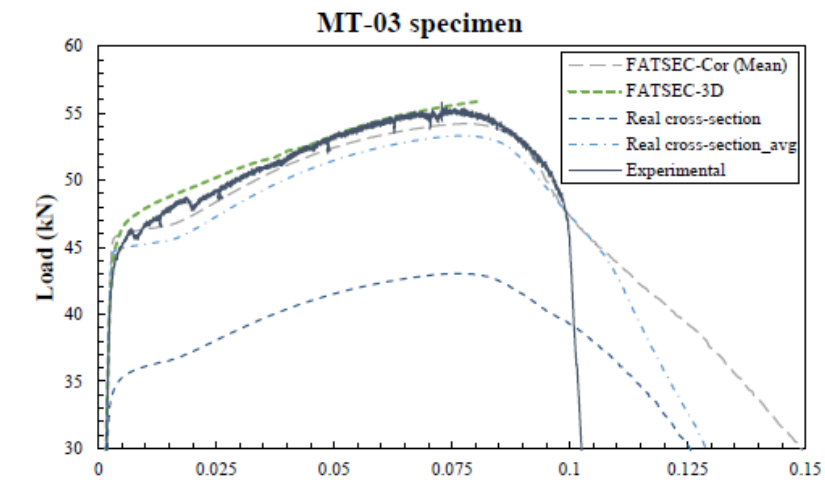

\section{Conclusions}

The following conclusion can be drawn from the presented work.

1) 3D scan technique is presented as a useful tool with many different applications. The description of the pitting distribution and the definition of the critical cross-section is possible with this kind of methods. In addition, the evaluation of the corrosion distribution along the specimen is possible by obtaining the cross-section reduction along the scanned bar.

2) 3D scan post-process re-meshing to transform the triangular surface mesh into a solid tetrahedral mesh is not straightforward, and presents some technical difficulties. Spikes and holes on the surface mesh are difficult to detect and remove. Moreover, complex shapes due to pitting makes it difficult to convert the surface mesh into nurb surfaces or solids. Therefore, the definition of the pits has to be accurately enough to describe the local effect produced into the bar due to the 3D multi-axial state so a compromise between accuracy in the pit description and number of elements has to be found.

3) The presented model allows reproducing the fatigue life and tensile behaviour with a very good agreement with respect to the experimental data. Fatigue life estimation require a high computational cost since in each load step the non-linear stress state has to be obtained. On the other hand, tensile tests require low computational cost even though many intermediate steps to describe the $\sigma-\varepsilon$ behaviour until ultimate bar capacity are needed.

4) The geometry of both corroded bars and un-corroded ribbed bars induce complex stress distribution and 3D stress states. The material model and heterogeneity description 
used for the description of TEMPCORE ${ }^{\circledR}$ bars adjusted very well for both situations; hence indicating that the heterogeneous distribution of elasto-plastic materials is adequate for the description of estimation the maximum capacity. However, it should be mentioned that the softening response was not well captured, which might be relevant in some ductility applications. Further research is needed in this respect, in order to better capture the post peak response.

5) A multi-axial stress state takes place in the critical pitted cross-section. Simpler uniaxial models require an overestimation of the steel critical cross-section area to include these effects and get a good estimation of the yielding and ultimate stresses, to describe the overall behaviour properly. The higher corrosion, the larger difference observed between the actual 3D geometry and that of single pit idealization

6) The variation of the mechanical properties observed from the experimental test can be well described by means of mechanical effects that corrosion produces on the bar due to pit distribution over the steel surface. 3D models results are good to describe with a very good detail the different mechanical phenomena observed in the specimens of this experimental campaign.

\section{Acknowledgments}

The authors wish to acknowledge the financial support of The Ministry of Economy and Competitiveness of the Government of Spain (MINECO) for providing funds for projects BIA2009-11764, BIA2012-36848 and the European Regional Development Funds (ERDF). The financial support of Infrastructures de Catalunya (ICAT) is also highly appreciated. Acknowledgements are also extended to CIMNE to providing a license of the software $\mathrm{GiD}$ necessary to develop the presented work.

\section{Appendix A}




\section{References}

[1] K. Tutti, Corrosion of steel in concrete, (1997) 6-15. doi:10.4324/9780203414606_chapter_2.

[2] L. Bertolini, B. Elsener, P. Pedeferri, R. Polder, Corrosion of Steel in Concrete. Prevention, Diagnosis, Repair., Wiley-VCH Verlag GmbH \& Co. KGaA, Weinheim, Germany, 2004.

[3] J. Broomfield, Corrosion of steel in concrete: understanding, investigation and repair, 2nd Ed., Taylor \& Francis, Abingdon, United Kingdom, 2002.

[4] M. Raupach, B. Elsener, R. Polder, J. Mietz, Corrosion of reinforcement in concrete, Woodhead Publishing Limited, 2007. doi:10.1533/9781845692285.

[5] W. Zhang, X. Song, X. Gu, S. Li, Tensile and fatigue behavior of corroded rebars, Constr. Build. Mater. 34 (2012) 409-417. doi:10.1016/j.conbuildmat.2012.02.071.

[6] C.A. Apostolopoulos, Mechanical behavior of corroded reinforcing steel bars S500s tempcore under low cycle fatigue, Constr. Build. Mater. 21 (2007) 1447-1456. doi:10.1016/j.conbuildmat.2006.07.008.

[7] C.A. a. Apostolopoulos, V.G.G. Papadakis, Consequences of steel corrosion on the ductility properties of reinforcement bar, Constr. Build. Mater. 22 (2008) 2316-2324. doi:10.1016/j.conbuildmat.2007.10.006.

[8] F. Tang, Z. Lin, G. Chen, W. Yi, Three-dimensional corrosion pit measurement and statistical mechanical degradation analysis of deformed steel bars subjected to accelerated corrosion, Constr. Build. Mater. $70 \quad$ (2014) 104-117. doi:10.1016/j.conbuildmat.2014.08.001.

[9] I. Fernandez, J.M. Bairán, A.R. Marí, Corrosion effects on the mechanical properties of reinforcing steel bars. Fatigue and $\sigma-\varepsilon$ behavior, Constr. Build. Mater. 101 (2015) 772783. doi:10.1016/j.conbuildmat.2015.10.139.

[10] I. Fernandez, J. M. Birán, A. R. Marí, Mechanical model to evaluate steel reinforcement corrosion effects on $\sigma-\varepsilon$ and fatigue curves. Experimental calibration and validation, Eng. Struct. (2016). http://dx.doi.org/10.1016/j.engstruct.2016.03.055.

[11] C.A. Apostolopoulos, S. Demis, V.G. Papadakis, Chloride-induced corrosion of steel reinforcement - Mechanical performance and pit depth analysis, Constr. Build. Mater. 38 (2013) 139-146. doi:10.1016/j.conbuildmat.2012.07.087.

[12] C.A. Apostolopoulos, G. Diamantogiannis, A.C. Apostolopoulos, Assessment of the Mechanical Behavior in Dual-Phase Steel B 400 C , B 450 C , and B 500 B in a Marine Environment, 28 (2016) 1-9. doi:10.1061/(ASCE)MT.1943-5533.0001271.

[13] M.M. Kashani, A.J. Crewe, N.A. Alexander, Use of a 3D optical measurement technique for stochastic corrosion pattern analysis of reinforcing bars subjected to accelerated corrosion, Corros. Sci. 73 (2013) 208-221. doi:10.1016/j.corsci.2013.03.037.

[14] G. Mancini, F. Tondolo, L. Iuliano, P. Minetola, Local reinforcing bar damage in r.c. members due to accelerated corrosion and loading, Constr. Build. Mater. 69 (2014) 116123. doi:10.1016/j.conbuildmat.2014.07.011.

[15] X. Wang, W. Zhang, X. Gu, H. Dai, Determination of residual cross-sectional areas of corroded bars in reinforced concrete structures using easy-to-measure variables, Constr. Build. Mater. 38 (2013) 846-853. doi:10.1016/j.conbuildmat.2012.09.060. 
[16] L. Llano Trueba, Patterns of corroded rebar surfaces and their impact on tensile mechanical properties., University of Surrey, 2015. http://epubs.surrey.ac.uk/808602/.

[17] I. Fernandez, M.F. Herrador, A.R. Marí, J.M. Bairán, Structural effects of steel reinforcement corrosion on statically indeterminate reinforced concrete members, Mater. Struct. (2016). doi:10.1617/s11527-016-0836-2.

[18] Diana, TNO Diana. Finite Element Analysis User’s Manual - release 9.6.6: TNO, Delft, Netherlands, 2015.

[19] C.I.C. for N.M. in Engineering, GID. The personal pre and post processor, (2014).

[20] T.E.A. El Maaddawy, K.K.A. Soudki, Effectiveness of impressed current technique to simulate corrosion of steel reinforcement in concrete, J. Mater. Civ. .... (2003) 41-47. http://ascelibrary.org/doi/abs/10.1061/(ASCE)0899-1561(2003)15:1(41) (accessed July 3, 2014).

[21] M. Badawi, K. Soudki, Control of Corrosion-Induced Damage in Reinforced Concrete Beams Using Carbon Fiber-Reinforced Polymer Laminates, J. Compos. Constr. 9 (2005) 195-201. doi:10.1061/(ASCE)1090-0268(2005)9:2(195).

[22] M. Saifullah, L.L.A.A. Clark, M. Sailfullah, L.L.A.A. Clark, Effect of Corrosion Rate on the Bond Strength of Corroded Reinforcement, in: S.A. Press (Ed.), Proc. Int. Conf. Corros. Corros. Prot. Steel Concr., University of Sheffield, 1994: pp. 591-600.

[23] UNE36065:2011, Ribbed bars of weldable steel with special characteristics of ductility for the reinforcement of concrete, (2011).

[24] ASTM Standard G1, Standard practice for preparing, cleaning, and evaluating corrosion test specimens, (2011).

[25] 3D Systems, Geomagic Wrap 2014, Http://www.geomagic.com/en/. (n.d.).

[26] UNE-EN-ISO-15630-01, Steel for reinforced and prestresed concrete - Testing methods. Part 1: Bars and wires for reinforced concrete, (n.d.).

[27] Eurocode 2: Design of concrete structures - EN1992, (1992).

[28] Eurocode 3: Design of steel structures - EN 1993, (1993).

[29] J.M. Bairan, A.R. Marí, H. Ortega, J.C. Rosa, Effects of winding and straightening of medium and large diameter reinforcing bars manufactured in coils in their mechanical properties, Mater. Construcción. 61 (2011) 559-581. doi:10.3989/mc.2011.60110. 